\title{
Long Term Effect of Biodegradable Polymer on Oxidative Stress and Genotoxicity
}

\author{
Divya Surendran, Sarath Kumar R.S., Geetha C.S., Mohanan P.V.*
}

Toxicology Division, Biomedical Technology Wing, Sree Chitra Tirunal Institute for Medical Sciences and Technology, Thiruvananthapuram - 695 012, Kerala, India.

*Corresponding Author: Mohanan P.V, Toxicology Division, Biomedical Technology Wing, Sree Chitra Tirunal Institute for Medical Sciences and Technology, Thiruvananthapuram - 695 012, Kerala, India.

Telephone number-91471-2520266; Email: mohanpv10@gmail.com; mohanpv@sctimst.ac.in

doi:10.5618/bio.2012.v2.n1.4 || Received: 04-05-2012, Accepted: 19-06-2012, Available online: 21-06-2012

\begin{abstract}
Oxidative stress and genotoxicity induced by intraperitoneally implanted polycaprolactone (PCL) were evaluated in present study. In an effort to assess oxidative stress induced liver injury, we measured hematological indices, biochemical parameters of liver function and levels of oxidative stress biomarkers such as lipid peroxidation (LPO), 8hydroxy-2'deoxy guanosine (8-OHdG), reduced glutathione(GSH) and glutathione reductase(GR) after three months as well as twelve months of implantation. Another objective of the study was to analyze chromosomal aberrations induced by the extract of polycaprolactone in human peripheral venous blood lymphocytes using GTG banding technique and karyotyping of human chromosomes. No significant changes were displayed by the experimental animals in the hematological parameters. Furthermore, liver function were normal as evidenced by the activities of aspartate amino transferase (AST), alanine amino transferase (ALT), alkaline phosphatase (ALP), serum bilirubin, cholesterol, triglyceride and albumin. Oxidative stress statuses were comparable with the control animals after 3 and 12 months of intraperitoneal implantation. Karyotype of human chromosomes were found to be normal indicating the nongenotoxic effects of polycaprolactone. Hence it can be concluded that polycaprolactone used for this study is non-toxic at the molecular level, under the environmental conditions monitored.
\end{abstract}

Keywords: polycaprolactone side effects; genotoxicity; oxidative stress; GTG banding; 8-hydroxy-2'deoxy guanosine; antioxidant

\section{Introduction}

Polycaprolactone (PCL) is a biodegradable polyester with low melting point. The most common use of PCL is in the manufacture of speciality polyurethanes. PCL has been approved by the FDA in specific applications used in the human body as a drug delivery device, suture or adhesion barrier. It is being investigated as a scaffold for tissue repair via tissue engineering and GBR membrane. It is also used in a variety of drugs have been encapsulated within PCL beads for controlled release and targeted drug delivery.

Long term biocompatibility of implanted biomaterials can be evaluated by determining the oxidative stress and genotoxic effects induced by the polymers. Peritoneal cavity provides an ideal environment [1] to analyze the toxicity of biodegradable synthetic polyesters such as polycaprolactone which is an attractive candidate for various medical applications especially drug delivery [2]. When implanted foreign materials come in contact with blood, they may bring about changes in hematological indices, an important parameter which gives us information on the physiological status of animals and humans [3]. Implanted biodegradable synthetic polyesters disintegrate into constituents which are resorbed by the body and metabolized via citric acid cycle. [4]. The leachants may enter the circulation and produce adverse effects on different organs [5] especially liver, a major site where endogenous and exogenous substances accumulate. Free radicals are generated [6], during the detoxification of reactive metabolites, accomplished by cytochrome P-450, located in the smooth endoplasmic reticulum of hepatocytes [7] and also by NADPH oxidase enzyme [8] in the activated Kupffer cells [9]. The reactive species when elevated above a threshold level oxidizes polyunsaturated fatty acids resulting in the onset of lipid 
peroxidation [10] giving rise to peroxyl and alkoxyl radicals, the primary end products. Malondialdehyde is one of the toxic secondary product of lipid peroxidation which can diffuse and mutilate cellular constituents such as lipids, nucleic acids and proteins by their electrophilic nature leading to cell death [11].

Hydroxyl radicals interact with DNA leading to the oxidation of guanine resulting in $8-\mathrm{OHdG}$, a mutagenic lesion. 8-oxoguanine DNA glycosylase-1 (OGG1) is a major DNA repair enzyme of the base excision repair (BER) pathway which play an important role in the removal of these DNA adducts formed. If not repaired before replication, $8 \mathrm{OHdG}$ will lead to $\mathrm{G}$ to T DNA transversions [12]. In order to quantify 8-OHdG, ELISA is found to be a convenient method. [13].

Liver is well equipped with antioxidant defense system, which scavenges free radicals and thus safeguards cells from damage [14]. Hepatocytes are the storehouse of glutathione, an electron rich tripeptide synthesized, utilized and regenerated via gammaglutamyl cycle. The nucleophilic property of the sulphydryl group present in GSH assist in scavenging free radicals and its reducing power aids in recycling other oxidized antioxidants [15]. GR is a homodimeric flavoprotein which utilizes NADPH to convert oxidized GSSG to GSH. [16].

Genotoxicity evaluation is an essential part of toxicity studies. In vitro human peripheral blood lymphocyte culture serves as a suitable test system to reveal the genotoxic potential of polycaprolactone [17].Cytogenetic effects of Polycaprolactone via G banding technique have not been extensively studied. $\mathrm{G}$ banding of human chromosomes facilitate the identification of individual chromosomes and help to localize sites of gross abnormalities and abnormal structures on the basis of banding patterns [18].

The current study was undertaken to evaluate the effect of intraperitoneally implanted polycaprolactone and polyglactin (biodegradable polymers), in vivo, on rat liver glutathione reductase and reduced glutathione, lipid peroxidation, hematological and biochemical response along with the evaluation of oxidative DNA damage using 8 -OHdG as the biomarker were performed. This study was conducted to also assess the cytocompatability of polycaprolactone extract on human lymphocytes.

\section{Materials and Methods}

2.1 Materials. Polycaprolactone and polyglactin were procured from the Dental Products Laboratory, Biomedical Technology Wing, Sree Chitra Tirunal Institute for Medical Sciences and Technology,
Trivandrum, Kerala, India. Thiobarbituric acid (TBA), reduced glutathione (GSH), Oxidized glutathione (GSSG), Dithio-bis-2-nitrobenzoic acid (DTNB), Cyclophosphamide, S9 from liver pooled, Demecolchicine, Giemsa stain were purchased from Sigma Aldrich (St. Louis, MO, USA). Peripheral blood karyotyping medium, Trypsin-EDTA from Kibbutz beit Haemek (Israel). RPMI-1640 was obtained from Himedia (India). The other chemicals and agents used were of analytical grade.

2.2 Animals. Wistar rats (120g body weight, Division of Lab Animal Science, Sree Chitra Tirunal Institute for Medical Sciences and Technology) were housed in individual polypropylene fabricated cages with stainless steel top mesh placed in a temperature-controlled room. Room temperature was maintained at $22 \pm 3^{\circ} \mathrm{C}$, relative humidity $30-70 \%$ and a $12 \mathrm{hr}$ light/dark cycle. Distilled water and standard pellet diet were supplied ad libitum to the animals. They were acclimatized to the environment five days prior to the initiation of experiment. All procedures in animal experiments were in compliance with the guidelines of Institute of Animal Ethics Committee regulations approved by Committee for the Purpose of Control and Supervision of Experiments on Animals.

2.3 Intraperitoneal implantation. 8 week old animals were divided into two groups (6 rats per group): group 1 (rats implanted with polyglactin as control material), group 2 (rats implanted with polycaprolactone). Two sets of these groups were taken. One set each was kept for an observation period of 3months and another for 12 months. All animals were anesthetized by means of intraperitoneal injection of ketamine hydrochloride 100 $\mathrm{mg} / \mathrm{kg}$ (Levent, Istanbul, Turkey) and xylazine $5 \mathrm{mg} / \mathrm{kg}$ (Bayer, Istanbul, Turkey).

A $2 \mathrm{~cm}$ median laprotomy was performed. $20 \mathrm{~mm}$ length $\mathrm{x} 0.25 \mathrm{~mm}$ diameter polyglactin and $20 \mathrm{~mm}$ length x $0.34 \mathrm{~mm}$ thick $\times 0.5 \mathrm{~mm}$ width polycaprolactone was implanted into the intraperitoneal cavity of group1 and group 2 rats. The abdominal wall and skin were then sutured with absorbable and non absorbable surgical sutures (Ethicon, Sommerville, NJ, USA). The symptom and mortality were observed and recorded carefully everyday for 3 months and 12 months. At the end of the experimental period, blood was collected in vials containing EDTA from orbital sinus after being anesthetized by ether for haematological analysis and then sacrificed. Serum was collected by centrifuging blood at 2500rpm for $10 \mathrm{~min}$ for biochemical analysis. The rat liver was excised and washed with $0.9 \%$ cold saline and stored (for DNA extraction) in $-20^{\circ} \mathrm{C}$ until use. 
2.4 Determination of Biochemical parameters. Liver function was assessed with the serum levels of alanine aminotransferase (ALT) alkaline phosphatase (ALP), aspartate aminotransferase (AST), Gamma-glutamyl transferase (GGT), total protein (TP), albumin (ALB), total bilirubin (TBIL), triglycerides(TG), total cholesterol (TCHO) using the commercial kits (Buhlmann Laboratories, Switzerland). All biochemical assays were performed using a clinical automatic chemistry analyzer ERBA Manheim XL- 300 Transasia (Germany).

2.5 Determination of Haematological parameters. Blood samples were collected in vials containing EDTA as anticoagulant. Red blood cells (RBC), white blood cells (WBC), hemoglobin (HGB), platelets (PLT), mean cell volume (MCV), mean cell hemoglobin concentration (MCHC), and hematocrit (HCT) were measured using a ABX Micros OT Automated Hematology Analyzer.

2.6 Estimation of lipid peroxidation. Level of lipid peroxides (LPO) in liver was determined as the concentration of malonialdehyde (MDA) generated by thiobarbituric acid (TBA) reaction to form a pink colored complex. The end product MDA produced, was determined with the absorbance coefficient of the MDA-TBA complex at $532 \mathrm{~nm}$ in spectrophotometer [19].

2.7 Determination of Reduced Glutathione, Glutathione reductase and Protein. In order to determine the GSH level, a sub sample of tissue was thawed, and homogenized using $0.1 \mathrm{M}$ phosphate buffer. GSH contents were estimated by Ellman's reaction using DTNB as described elsewhere [20] with slight modification [21].

The activity of glutathione reductase (GR) was assayed by measuring the reduction of GSSG in the presence of NADPH [22]. The content of protein in the liver homogenate was determined following Lowry et al. (1951) method. [23].

2.8 DNA Isolation and determination of 80HdG in rat liver. DNA isolation was carried out using Gen Elute Mammalian Genomic DNA isolation kit (Sigma Aldrich) according to manufactures instructions .Samples containing 50ug of DNA were resuspended in $50 \mathrm{ug}$ of reaction mixture, containing $100 \mathrm{mmol} / \mathrm{l}$ sodium acetate $\left(\mathrm{pH}\right.$ 5.0) and $5 \mathrm{mmol} / \mathrm{l} \mathrm{MgCl}_{2}$, and digested with $1 \mu \mathrm{l}$ of DNase I, for $10 \mathrm{~min}$ at room temperature. The amount of 8-OHdG was determined in liver DNA digests using 8-OHdG-ELISA CHECK Kit (Qiagen) a. competitive ELISA method. DNA-digested samples were added to the micro titer plate pre coated with 8-OHdG, and the assay was performed according to the manufacturer's instructions.

2.9 Chromosomal aberrations assay. 1) Preparation of material extract and peripheral blood lymphocyte culture: Material extract preparation and peripheral blood lymphocyte culture was performed as described elsewhere [21] with slight modification. Cyclophosphamide (100 $\mu \mathrm{g} /$ culture) alone was used as positive control with and without the metabolic activator S9. The cultures were treated with spindle poison colchicine (final concentration of $1 \mu \mathrm{g} /$ culture) for half an hour and cells were harvested at $69 \mathrm{hr}$.

2) Chromosome harvest and slide preparation: Chromosome harvest and slide preparation were carried out as described by B. Lakshmi Sowjanya et al [24] with slight modification. The centrifugation steps were carried out at $2000 \mathrm{rpm}$ for $10 \mathrm{~min}$. After decanting the supernatant carefully, $0.075 \mathrm{M} \mathrm{KCL}$ was added very slowly to the pellet by vortexing the tubes. Freshly prepared 3:1 methanol: acetic acid was used as fixative at room temperature instead of ice cold preparation.

3) G banding using trypsin and Giemsa stain (GTG banding) and Karyotyping: Metaphase slides were used for GTG banding 3-4 days after slide preparation. The dry slides were kept at $90{ }^{\circ} \mathrm{C}$ oven and baked for 60 minutes and cooled followed by treatment with $0.025 \%$ trypsin -EDTA for 10-25 seconds. The reaction was stopped by treating slides with $0.9 \%$ saline. Cells were stained in $4 \%(\mathrm{v} / \mathrm{v})$ Giemsa stain in Sorenson buffer (pH6.8) for 10- 15 minutes. The slides were rinsed in distilled water, dried, mounted using Disterene Plasticizer Xylene (DPX) and then karyotyped for analyzing chromosomal aberrations using automated metaphase finder (Carl Zeiss, Germany) and photographed.

2.10 Statistical analysis. The results were expressed as mean \pm S.D. The total variation and difference among means were analyzed through student $\mathrm{t}$ test and the $p$ value less than or equal to 0.05 were considered significant.

\section{Results and Discussion}

In the present study, we made an attempt to determine whether intraperitoneal implantation of polycaprolactone resulted in liver damage due to oxidative stress and genotoxicity in human peripheral blood lymphocytes during implantation period of 3 months and 12 months.

Table 1 exhibits the changes in hematological indices of rats after 3 and 12 months of PCL implantation. Results indicate that WBC, RBC, HGB of 
polycaprolactone implanted wistar rats were not significantly reduced after 12 months of implantation $(\mathrm{p} \geq 0.05)$, while PLT, MCV, MCHC were slightly elevated $(p \geq 0.05)$ when compared to short term implanted group(Table 1). As there is no marked changes in RBC, WBC, HGB the implanted polymers seems to have little influence in inducing anemia in the experimental rats. Decrease in leucocytes may be due to phagocytosis of particulate debris of the polymers intraperitoneally; releasing free radicals, cytokines, degradative enzymes. Increase of platelets show the possible effect of the implanted polyesters on blood coagulation causing damage to platelets but not to significant levels.

Table 1. Effect of PCL on Hematological parameters after 3 and 12 months of intraperitoneal implantation in Wistar rats.

\begin{tabular}{llllc}
\hline \multirow{2}{*}{$\begin{array}{c}\text { Hematological } \\
\text { parameters }\end{array}$} & \multicolumn{2}{c}{ Sub chronic toxicity $^{\mathbf{c}}$} & \multicolumn{2}{c}{ Chronic toxicity $^{\mathbf{d}}$} \\
\cline { 2 - 5 } & \multicolumn{1}{c}{ Control $^{\text {a }}$} & \multicolumn{1}{c}{ Test $^{\mathbf{b}}$} & \multicolumn{1}{c}{ Control $^{\text {a }}$} & Test $^{\mathbf{b}}$ \\
\hline Platelet $\times 10^{3} / \mathrm{mm}^{3}$ & $825.67 \pm 319.10$ & $1027.33 \pm 143.58$ & $1011.67 \pm 144.32$ & $1030.83 \pm 113.07$ \\
$\mathrm{WBC} \times 10^{3} / \mathrm{mm}^{3}$ & $9.23 \pm 1.95$ & $8.7 \pm 1.67$ & $6.18 \pm 2.17$ & $6.17 \pm 1.94$ \\
$\mathrm{RBC} \times 10^{6} / \mathrm{mm}^{3}$ & $8.78 \pm 0.79$ & $9.18 \pm 0.85$ & $8.83 \pm 0.99$ & $8.33 \pm 0.97$ \\
$\mathrm{Hb} \mathrm{g} / \mathrm{dl}$ & $15.58 \pm 1.22$ & $16.9 \pm 0.77$ & $16.25 \pm 1.18$ & $15.42 \pm 1.46$ \\
$\mathrm{MCV} \mu \mathrm{m}^{3}$ & $51.5 \pm 2.57$ & $51.83 \pm 2.64$ & $53.17 \pm 2.66$ & $51.17 \pm 2.23$ \\
$\mathrm{MCHC} \mathrm{g/dl}$ & $34.58 \pm 0.64$ & $35.3 \pm 0.47$ & $35.03 \pm 2.11$ & $36.35 \pm 2.55$ \\
\hline
\end{tabular}

${ }^{\mathrm{a}}$ Control- Rats implanted with polyglactin, ${ }^{\mathrm{b}}$ Test - Rats implanted with polycaprolactone,

${ }^{\mathbf{c}}$ Sub chronic toxicity- after 3 months of implantation, ${ }^{\mathbf{d}}$ Chronic toxicity- after 12 months of implantation. Values are expressed as mean \pm S.D., $n=6, p \leq 0.05$

The serum biochemical parameters were assayed further evaluate the toxicity of intraperitoneally implanted PCL and is presented in (Table 2). Rats implanted with PCL and PGL showed decrease in AST, ALT and increase in ALP after 12 months compared to their respective control groups and also between 3 and 12 months of PCL implantation. Reasons for this could be increase in the age of rats [25], metabolic changes due to decomposition of the material. On the other hand ALT/AST ratio was found to be less than 1 indicating absence of inflammatory condition or liver damage [26].

Serum bilirubin and protein concentration also showed insignificant reduction suggesting that the increase in age has not impaired the gluconyl transferase system or has effected bilirubin elimination and absence of liver dysfunction due to changes in protein levels. The risk of oxidative damage increases with higher lipid concentration. [27]. Raised serum triglyceride and cholesterol levels were seen after 12 months of implantation but not to significant levels probably reflecting age related changes and absence of oxidative damage. Proper functioning of kidneys can be determined by measuring serum creatinine and urea. In our results there were only slight changes in long term implanted rats which suggests normal kidney functioning. Furthermore, drop in albumin is an indicator of inflammation. In our findings, none of the implanted rats showed significant changes in albumin levels.

Hepatic GSH was measured in rats after 3 and 12 months of implantation and its levels in control and test group are presented in Figure 1. GSH concentration in control liver was amounted to $6.7 \mathrm{nM} / \mathrm{mg}$ protein after 3 months of implantation while in experimental rats implanted with PCL the liver GSH amounted to7.1 $\mathrm{nM} / \mathrm{mg}$ protein $(\mathrm{p} \geq 0.05)$. However in subsequent group the liver GSH were found to decrease to $3.68 \mathrm{nM} / \mathrm{mg}$ tissue and $3.55 \mathrm{nM} / \mathrm{mg}$ tissue in control and test. The activity of GR is demonstrated in Figure 2. Mean GR activity for the liver was high in long term PCL implanted control and experimental rats when compared to their counterpart and amounted to 0.37 Units/mg protein and 0.4 Units/mg protein. The effect of intraperitoneally implanted PCL on the levels of MDA content is depicted in Figure 3. In short term implantation of PCL, the level of LPO in liver was $3.01 \mathrm{~nm} / \mathrm{mg}$ and $2.78 \mathrm{~nm} / \mathrm{mg}$ in control and test group. The values correlated well with long term implanted 
control and test groups and were found to be $2.69 \mathrm{~nm} / \mathrm{mg}$ and $2.73 \mathrm{~nm} / \mathrm{mg}$.

Table 2. Effect of PCL on Serum biochemical parameters after 3 and 12 months of intraperitoneal implantation in Wistar rats.

\begin{tabular}{lllll}
\hline \multirow{2}{*}{$\begin{array}{c}\text { Biochemical } \\
\text { parameter }\end{array}$} & \multicolumn{2}{c}{ 90days toxicity } & \multicolumn{2}{c}{ 12 months toxicity } \\
\cline { 2 - 5 } & \multicolumn{1}{c}{ Control $^{\text {a }}$} & \multicolumn{1}{c}{ Test $^{\mathbf{b}}$} & \multicolumn{1}{c}{ Control $^{\text {a }}$} & \multicolumn{1}{c}{ Test $^{\mathbf{b}}$} \\
\hline ALT & $127.1 \pm 17.08$ & $116.25 \pm 51.77$ & $125.4 \pm 27.57$ & $110.6 \pm 22.78$ \\
AST & $206.88 \pm 57.78$ & $169.47 \pm 28.76$ & $141.8 \pm 40.42$ & $138.92 \pm 37.89$ \\
ALP & $201.5 \pm 45.58$ & $205.47 \pm 24.32$ & $230.5 \pm 62.75$ & $239.5 \pm 36.02$ \\
Total Protein & $8.53 \pm 0.46$ & $8.21 \pm 0.81$ & $8.78 \pm 0.58$ & $8.18 \pm 0.39$ \\
Creatinine & $1.12 \pm 0.13$ & $1.08 \pm 0.07$ & $1.01 \pm 0.06$ & $1.21 \pm 0.48$ \\
Urea & $42.53 \pm 2.44$ & $42.93 \pm 3.64$ & $43.35 \pm 4.78$ & $44.27 \pm 2.66$ \\
GGT & $17.03 \pm 2.97$ & $16.82 \pm 3.56$ & $17.93 \pm 3.54$ & $15.9 \pm 2.96$ \\
Glucose & $94.57 \pm 35.08$ & $95.63 \pm 11.96$ & $80.38 \pm 28.48$ & $82.15 \pm 11.02$ \\
Cholesterol & $80.17 \pm 5.78$ & $88.67 \pm 16.57$ & $105.5 \pm 22.47$ & $108.83 \pm 19.11$ \\
TAG & $194.67 \pm 98.80$ & $195.33 \pm 51.13$ & $197.33 \pm 41.08$ & $198.5 \pm 35.03$ \\
Total Bilirubin & $0.36 \pm 0.4$ & $0.32 \pm 0.5$ & $0.34 \pm 0.33$ & $0.27 \pm 0.22$ \\
Albumin & $4.43 \pm 0.46$ & $4.47 \pm 0.27$ & $4.5 \pm 0.45$ & $4.32 \pm 0.17$ \\
Calcium & $11.22 \pm 0.50$ & $11 \pm 0.41$ & $8.77 \pm 3.02$ & $9.07 \pm 3.33$ \\
Phosphate & $9.14 \pm 1.83$ & $7.52 \pm 0.85$ & $7.44 \pm 1.9$ & $7.15 \pm 0.81$ \\
Chloride & $143.85 \pm 16.44$ & $131.48 \pm 9.56$ & $129.42 \pm 1.87$ & $132.45 \pm 1.70$ \\
\hline
\end{tabular}

${ }^{\mathrm{a}}$ Control- Rats implanted with polyglactin, ${ }^{\mathrm{b}}$ Test - Rats implanted with polycaprolactone,

${ }^{\mathbf{c}}$ Sub chronic toxicity- after 3 months of implantation, ${ }^{\mathbf{d}}$ Chronic toxicity- after 12 months of implantation. Values are expressed as mean \pm S.D., $n=6, p \leq 0.05$

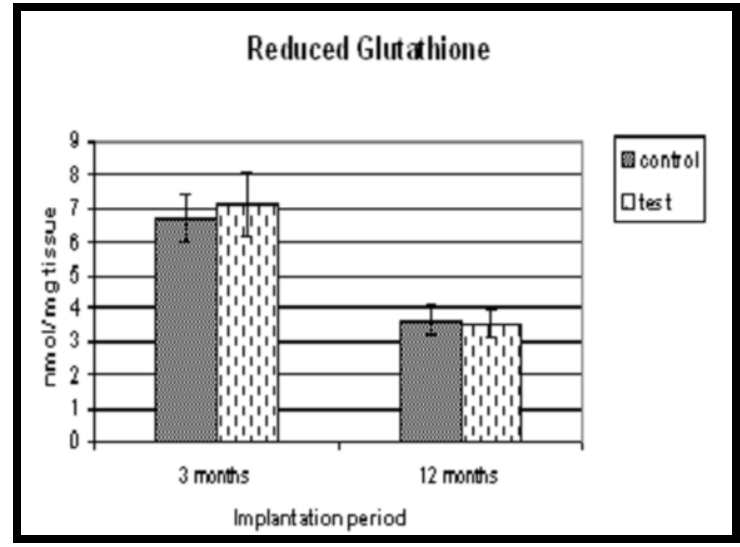

Figure 1. Effect of Polycaprolactone (PCL) on Reduced Glutathione in rat liver homogenate after 3 and 12 months of intraperitoneal implantation. (Values are expressed as mean \pm S.D., $n=6 . p \leq 0.05$ ).

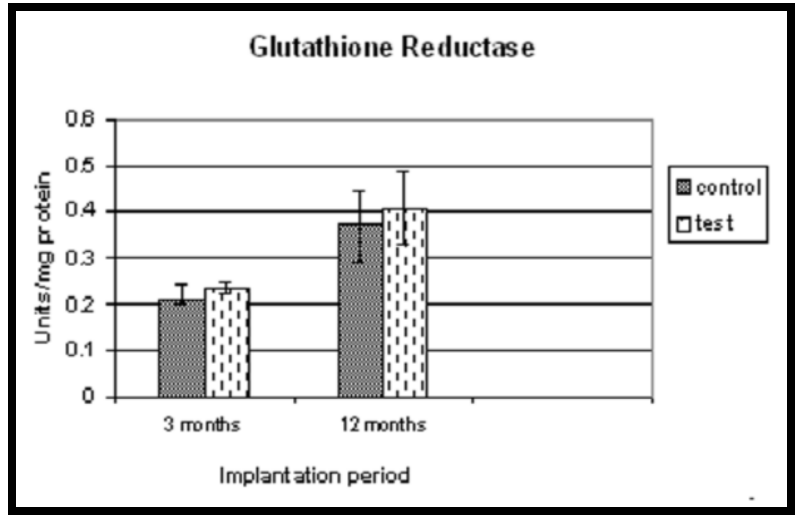

Figure 2. Effect of Polycaprolactone (PCL) on Glutathione reductase in rat liver homogenate after 3 and 12 months of intraperitoneal implantation. (Values are expressed as mean \pm S.D., $n=6 . p \leq 0.05$ ). 
The depletion of GSH accompanied by concomitant increase in GR activity and absence of significant LPO after 12 months of implantation compared to short term implanted group. This makes it clear that ageing process in rats and uptake of degraded particles of PCL by kupffer cells may be the factors that contributed towards free radical production. This brought substantial changes in reduced glutathione. GSH scavenges free radicals and play excellent role in protecting cell from LPO. However increase in GR activity and absence of LPO suggests that oxidative damage has not occurred to significant levels to initiate cytotoxic effects.

Figure 4 presents $8 \mathrm{OHdG}$ levels in liver after 3 and 12 months of PCL implantation. In Wistar rats, following short term implantation, the mean hepatic 8$\mathrm{OHdG}$ in control and test were $1.92 \mathrm{ng} / \mathrm{ml} / \mathrm{ug}$ and 2 $\mathrm{ng} / \mathrm{ml} / \mathrm{ug}$ respectively. However, in long term implanted rats $8 \mathrm{OHdG}$ levels were higher than their counterpart. Mean values were $3.91 \mathrm{ng} / \mathrm{ml} / \mathrm{ug}$ and $4.06 \mathrm{ng} / \mathrm{ml} / \mathrm{ug}$ in control and test groups. This can be explained by the interaction of free radicals generated during ageing process with DNA led to slight increment in $8 \mathrm{OHdG}$ levels after 12 months of implantation. Previous studies has shown that DNA polymerase $\beta$, a major enzyme of base excision repair becomes less efficient with age resulting in nuclear DNA damage and therefore increasing $8 \mathrm{OHdG}$ formation [12]. Slight elevation of $8 \mathrm{OHdG}$ levels in test animals after 3 months with respect to their control indicate that decomposition of polymer also contributed towards free radical production.

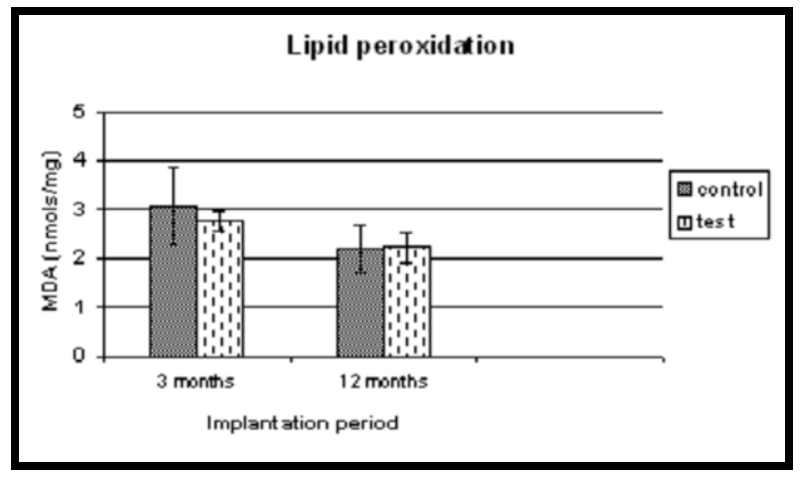

Fig 3. Effect of Polycaprolactone (PCL) on Lipid Peroxidation in rat liver homogenate after 3 and 12 months of intraperitoneal implantation (Values are expressed as mean \pm S.D., $n=6 . p \leq 0.05)$.

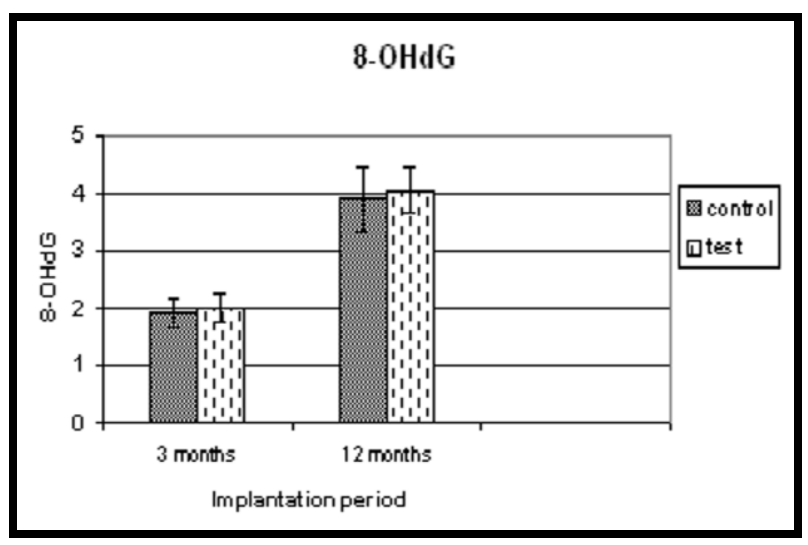

Fig 4. Effect of Polycaprolactone (PCL) on 8- hydroxyl 2 'deoxy guanosine $(8-\mathrm{OHdG})$ in rat liver homogenate after 3 and 12 months of intraperitoneal implantation. (Values are expressed as mean + S.D., $n=6 . p \leq 0.05$ ).

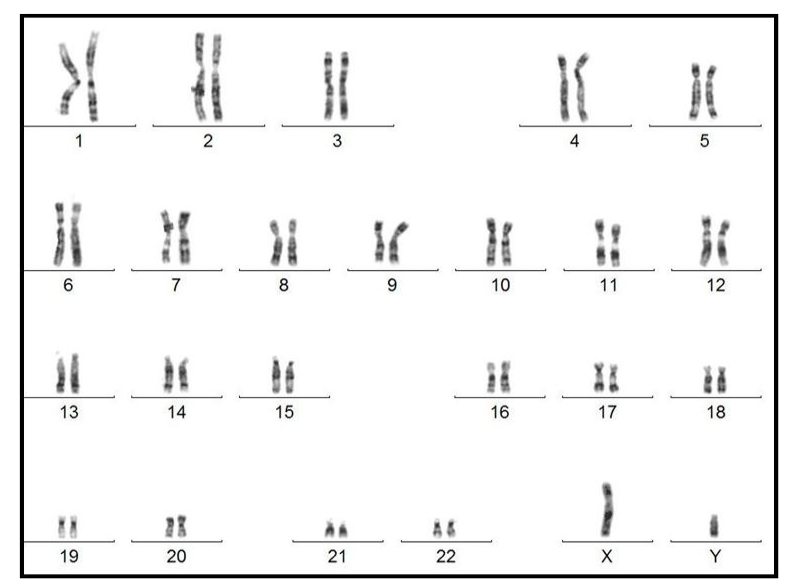

Fig 5 (A). Karyotype of GTG banded human chromosomes. Physiological saline treated (negative control).

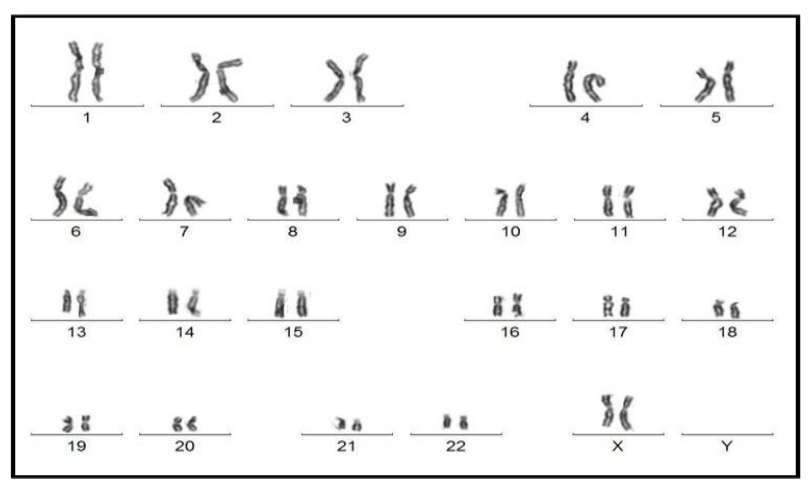

Fig 5 (B). Karyotype of GTG banded human chromosomes. Cyclophosphamide treated (Positive control). 


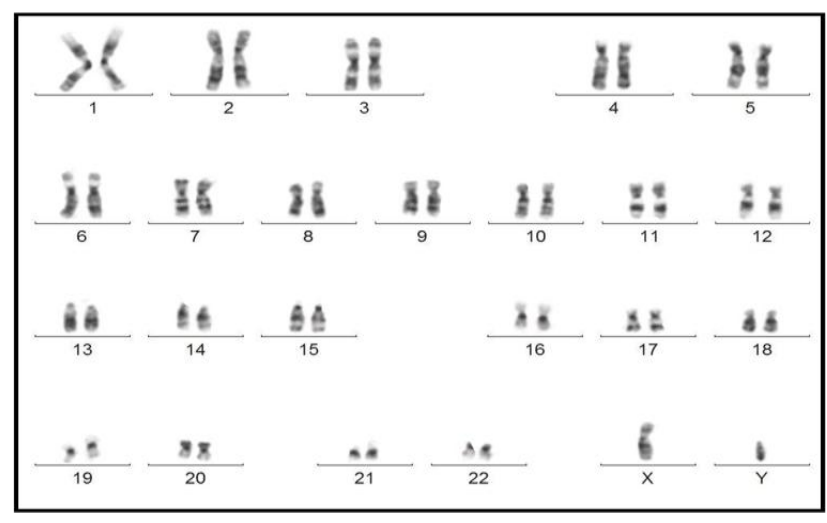

Fig 5 (C). Karyotype of GTG banded human chromosomes. Control- Polyglactin treated with S9.

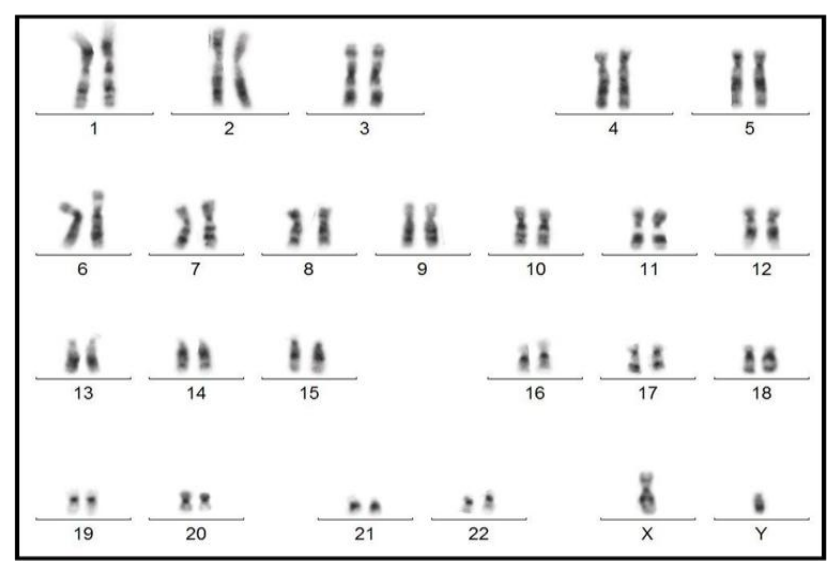

Fig 5 (D). Karyotype of GTG banded human chromosomes. Control-Polyglactin treated without S9.

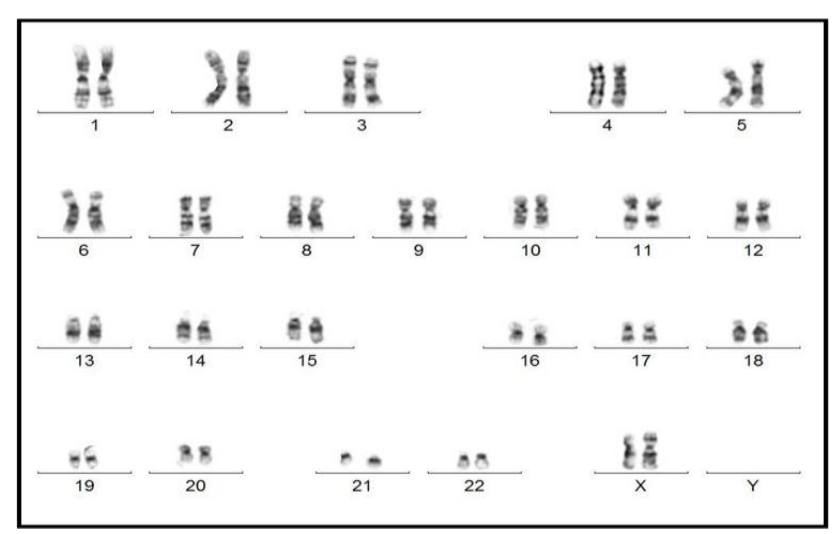

Fig 5 (E). Karyotype of GTG banded human chromosomes. Test- Polycaprolactone treated with S9.

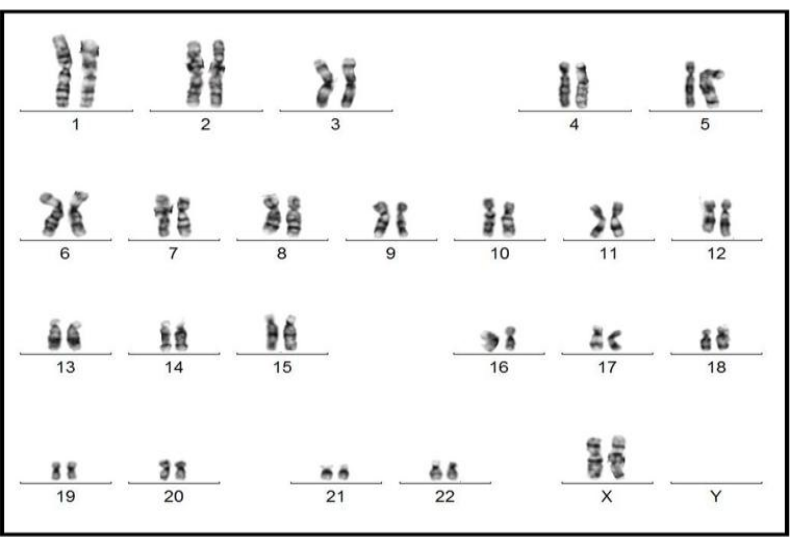

Fig 5 (F). Karyotype of GTG banded human chromosomes. Polycaprolactone treated without S9.

The results on the incidence of chromosomal aberration in in vitro lymphocytes induced by PCL and PGL extract and their negative and positive control are shown in Figure 5 (A, B, C, D, E, F) and Table 3. A low value was observed in both PGL and PCL treated with and without S9 compared with the values determined in cyclophosphamide treated lymphocytes. $(p \leq 0.05)$. The exposure of lymphocytes to PCL extract for $24 \mathrm{hr}$ did not induce any statistically significant aberration and correlated well with PGL treated cultures. $(\mathrm{p} \geq 0.05)$.A comparison of these values with negative control showed statistically insignificant results. ( $\mathrm{p} \geq 0.05$ ). In regard to specific types of structural aberrations studied using GTG banding of human chromosomes we obtained chromosome break at 46,XX,b(16)(q22), 46,XX,b(11)(q13.1), and structural changes in $12 \mathrm{q} 15$ and $7 \mathrm{q} 31.1$ in cyclophosphamide treated lymphocyte cultures, while normal karyotype was obtained in PCL and PGL treated lymphocytes and in negative control 46,XX. and 46,XY Figure 5 (A, B, $\mathrm{C}, \mathrm{D}, \mathrm{E}, \mathrm{F})$. In vitro studies give information on the toxicological effect of PCL and PGL in human blood samples. So far there few reports of the impact of PCL on lymphocytes cultures of humans [28] which suggests their non genotoxic effect and were consistent with the results. 
Table 3. Effect of physiological saline of PCL on in vitro human lymphocytes.

\begin{tabular}{|c|c|c|c|}
\hline \multirow{2}{*}{ Groups } & \multirow{2}{*}{$\begin{array}{l}\text { Number of } \\
\text { cells } \\
\text { Scored }\end{array}$} & \multicolumn{2}{|c|}{ Chromosomal aberrations } \\
\hline & & +S9 & $-\mathbf{S 9}$ \\
\hline $\begin{array}{l}\text { Cyclophosphamide (positive } \\
\text { control) }\end{array}$ & 100 & $93.00 \pm 5.67$ & - \\
\hline $\begin{array}{l}\text { Physiological saline } \\
\text { (negative control) }\end{array}$ & 100 & $3.5 \pm 0 \quad 0.71$ & $7.50 \pm 0.71$ \\
\hline Polycaprolactone & 100 & $9 \pm 2$ & $6.34 \pm 1.14$ \\
\hline Polyglactin & 100 & $8.74 \pm 1.41$ & - \\
\hline S9 alone & 100 & $3.00 \pm 1.41$ & - \\
\hline
\end{tabular}

a Metaphase scored from lymphocytes treated with Cyclophosphamide, physiological saline, polycaprolactone, polyglactin in the presence and absence of $S 9$ metabolic activator. Values are expressed as mean \pm S.D, $p \leq 0.05$.

Cyclophosphamide treated with the metabolic activator S9 served as positive control. S9 preparation is composed of microsomes and the microsomal membranes are enriched with drug metabolizing enzymes principally cytochrome P-450. The alkylating agent cyclophosphamide undergoes metabolic activation in the presence of cytochrome P450. Phosphoramide mustard is the ultimate cytotoxic metabolite through the intermediate agent hydroxyl cyclophosphamide and deschloroethyl cyclophosphamide [24] which is capable of inducing DNA strand lesions. Genotoxic effect of cyclophosphamide has been extensively studied in vitro and in vivo giving consistent positive results. Our results were consistent with the previous reports of [29] showing the site of chromosome breakage in most cases to be affected by $\mathrm{q}$ arm of medium sized chromosomes while short arm usually remained unaffected in cyclophosphamide treated chromosomes.

$\mathrm{G}$ banding of human chromosomes help to identify more precisely the chromosome segments involved in aberration. DNA- protein interactions are the important factors in banding studies. Trypsin treatment results in the degradation of non- histone proteins and differential distribution of chromatin produces $G$ bands. Dark G bands are known to be late replicating and are A-T rich and light $\mathrm{G}$ bands are early replicating and rich in $\mathrm{C}-\mathrm{G}$ pairs. [30].

\section{Conclusion}

In conclusion, we have successfully demonstrated the long term effect of intraperitoneally implanted PCL on hematological indices, biochemical parameters of liver function and levels of oxidative stress biomarkers such as lipid peroxidation (LPO), 8-hydroxy-2'deoxy guanosine (8-OHdG), reduced glutathione(GSH) and glutathione reductase(GR) after three months as well as twelve months of implantation. Alterations in hematological indices had little influence on haemostasis blood system. The data from serum biochemical parameters and oxidative stress biomarkers such as LPO, 8OHDG, GSH and GR indicated absence of oxidative stress induced liver injury. We also observed non-genotoxic effect of PCL extract in human lymphocytes in vitro via GTG banding and karyotyping. Hence it can be concluded that polycaprolactone used for this study is non-toxic at the molecular level, under the environmental conditions monitored.

\section{Acknowledgments}

Authors are thankful to the Director and Head BMT Wing, SCTIMST for providing the facilities to carry out the work. We are thankful to Dr. V Kalliyana Krishnan, Scientist-G, BMT Wing, SCTIMST for providing the materials. The authors gratefully acknowledge Ms. Lakshmi, Mr. S. Shaji and Mr. G. Harikumar for their support.

\section{References}

[1] Defrère, S.; Mestagdt, M.; Riva, R.; Krier, F.; Van L. A.; Drion, P.; Jérôme, C.; Evrard, B.; Dehoux, J.P.; Foidart, JM.; Donnez, J. In vivo biocompatibility of 
three potential intraperitoneal implants. Macromol Biosci., 2011, 11, 1336-1345.

http://dx.doi.org/10.1002/mabi.201100077

[2] Aishwarya, S.; Mahalakshmi, S.; Sehgal, P. K. Collagen-coated polycaprolactone microparticles as a controlled drug delivery system. $J$ microencapsul., 2008, 5, 298-306.

http://dx.doi.org/10.1080/02652040801972004

[3] Duan, Y.; Liu, J.; Ma, L.; Li, N.; Liu, H.; Wang, J.; Zheng, L.; Liu, C.; Wang, X.; Zhao, X.; Yan, J.; Wang, S.; Wang, H.; Zhang, X.; Hong, F. Toxicological characteristics of nanoparticulate anatase titanium dioxide in mice. Biomaterials., 2010, 31, 894-899. http://dx.doi. org/10.1016/j.biomaterials.2009.10.003

[4] James, M.; Anderson, A. R.; David, T. C. Foreign body reaction. Semin immunol. 2008, 2, 86-100.

[5] Mohanan, P.V.; Rathinam. K, Biocompatibility studies on silicone rubber. Proced. RC IEEE-EMBS and 14th BMESI. 1995, 4, 11-12.

[6] Dianzani, M.U. The role of free radicals in liver damage. Proceedings of the Nutrition Society. 1987, 46, 43-52. http://dx.doi.org/10.1079/PNS19870007

[7] Oesch, F.; Bentley, P.; Gopalan, M.; Stassiecki, P. Benzopyrene metabolism in subcellular fractions. Cancer research. 1985, 45, 4838-4843.

[8] Eloisa, A.; Manuel, C.; Alberto, M.; Francisco, S.; Consuelo, S. M. Decrease in free-radical production with age in rat peritoneal macrophages. Biochem. J. 1995, 312, 555-560.

[9] Wheeler, M. D.; Kono, H.; Yin, M.; Nakagami, M.; Uesugi, T.; Arteel, G. E.; Gäbele, E.; Rusyn, I.; Yamashina, S.; Froh, M.; Adachi, Y.; Iimuro, Y.; Bradford, B. U.; Smutney, O. M.; Connor, H. D.; Mason, R. P.; Goyert, S. M.; Peters, J. M.; Gonzalez, F. J.; Samulski, R. J.; Thurman, R.G. The role of Kupffer cell oxidant production in early ethanolinduced liver disease. Free radical Biology \& Medicine. 2001, 31, 1544-1549.

http://dx.doi.org/10.1016/50891-5849(01)00748-1

[10] Zacharias, E. S. Liposomal Antioxidants for Protection against Oxidant-Induced Damage. Journal of Toxicology. 2011, Article ID 152474:16 pages. DOI:10.1155/2011/152474.

[11] Jain, D.; Misra, R.; Kumar, A.; Jaiswal, G. Levels of malondialdehyde and antioxidants in the blood of patients with vitiligo of age group 11-20 years. Indian J Physiol Pharmacol 2008, 52, 297-301.

[12] Hashimoto, K.; Takasaki, W.; Sato, I.; Tsuda, S. DNA damage measured by comet assay and 80hdg formation related to blood chemical analysis in aged rats. J Toxicol Sci. 2007, 32, 249-259.

http://dx.doi.org/10.2131/its.32.249

[13] Akagi, S.; Nagake, Y.; Kasahara, J.; Sarai, A.; Kihara, T.; Morimoto, H.; Yano, A.; Nakao, K.; Nanba, K.; Ichikawa, H.; Makino, H. Significance of $8 \mathrm{OHdG}$ levels in patients with chronic renal failure. Nephrology. 2003, 8, 192-195.

http://dx.doi.org/10.1046/j.1440-1797.2003.00163.x

[14] Isabelle, A. L. Antioxidant defence mechanisms: new players in the pathogenesis of Non-alcoholic Steatohepatitis? Clinical Science. 2004, 106, 235237. http://dx.doi.org/10.1042/CS20030368

[15] Rocio, F.U.; Elias, F.; Jeanne, F.; Patrick, C.; Patrice, T. Evaluation of hepatic antioxidant systems after intravenous administration of polymeric nanoparticles. Biomaterials. 1997, 18, 511-517. http://dx.doi.org/10.1016/50142-9612(96)00178-0

[16] Cathcart, R.F. Vitamin C: the non toxic, non rate limited, antioxidant free scavenger. Med Hypotheses. 1985, 18, 61-77.

http://dx.doi.org/10.1016/0306-9877(85)90121-5

[17] Greil, J.; Spies, T.; Böswald, M.; Bechert, T.; Lugauer, S.; Regenfus, A.; Guggenbichler, J.P. Analysis of the acute cytotoxicity of the erlanger silver catheter. Infection. 1999, 27, S34-S37.

http://dx.doi.org/10.1007/BF02561615

[18] Feride, I. S.; Ahin, M.; Ali, E.; Erdal, T.; Adnan, M.E. The Mechanism of G-Banding Detected by Atomic Force Microscopy. Scanning. 1999, 22, $24-$ 27.

[19] Ohkawa, H.; Ohishi, N.; Yagi, K. Assay for lipid peroxides in animal tissues by thiobarbituric acid reaction. Anal Biochem. 1979, 95, 351-358. http://dx.doi.org/10.1016/0003-2697(79)90738-3

[20] Moron, M. S.; Depierre, J. W.; Mannervik, B. Levels of glutathione, glutathione reductase and glutathione $S$-transferase activities in rat lung and liver. Biochim Biophys Acta. 1979, 582, 67-78. http://dx.doi.org/10.1016/0304-4165(79)90289-7

[21] Arun, M.; Silja, P. K.; Mohanan, P.V. Evaluation of hydroxyapatite-bioglass and hydroxyapatite- ethyl vinyl acetate composite extracts on antioxidant defense mechanism and genotoxicity: An in vitro study. Toxicology Mechanisms and Methods. 2011, 21, 561-566. http://dx.doi.org/10.3109/15376516.2011.572933

[22] Mize, C. E.; Langdon, R. G. Hepatic glutathione reductase: purification and general kinetic properties. J Biol Chem.1962, 237, 1589-1595. 
[23] Lowry, O.H.; Rosérough, N. J.; Farr, A. L.; Randall, R. J. Protein measurement with the Folin phenol reagent. J Biol Chem. 1951, 193, 265-275.

[24] Lakshmi, S.B.; Rudrama, D. K.; Madhavi, D. Modulatory effects of garlic extract against the cyclophosphamide induced genotoxicity in human lymphocytes in vitro. Journal of Environmental Biology. 2009, 30, 663-666.

[25] Hilary, A. M.; Wassif, S. W.; Ian, W.; Dawn, A. S.; Karen, E. Age-Related Biomarkers can be modulated by Diet in the Rat. Food and Nutrition Sciences. 2011, 2, 884-890.

http://dx.doi.org/10.4236/fns.2011.28120

[26] Injac, R.; Perse, M.; Obermajer, N.; DjordjevicMilic, V.; Prijatelj, M.; Djordjevic, A.; Cerar, A.; Strukelj, B. Potential hepatoprotective effects of fullerenol $\mathrm{C} 60(\mathrm{OH}) \quad 24$ in doxorubicin-induced hepatotoxicity in rats with mammary carcinomas. Biomaterials. 2008, 29, 3451-3460.

http://dx.doi.org/10.1016/j.biomaterials.2008.04.048
[27] Balaraman, R.; Bafna, P.A.; Kolhapure, S. A. Antioxidant activity of dhc-1--a herbal formulation. Ethnopharmacol.. 2004, 94, 135-141.

http://dx.doi.org/10.1016/j.jep.2004.05.008

[28] Amelie, S.; Lotz, A.; Joachim, B.; Havlaa, E. R.; Katrin, F.; Rainer, S.; Rudolf, H.; Norbert, H. K. Cytotoxic And Genotoxic Effects Of Matrices For Cartilage Tissue Engineering. Toxicology Letters. 2009, 190, 128-133.

http://dx.doi.org/10.1016/j.toxlet.2009.06.880

[29] Matild, D.; Schuler, D. F. Cyclophosphamide induced chromosomal aberrations in non- tumorous patients. Humangenetik. 1974. 22, 221-227.

[30] Feride, I. S.; Ahin, M.; Ali, E.; Erdal, T.; Adnan, M.E. The Mechanism of G-Banding Detected by Atomic Force Microscopy. Scanning. 1999, 22, 2427. 